\title{
mCRPC: Systemische Sequenztherapie ist klinische Realität
}

- Prof. Peter Hammerer, Braunschweig, erklärte, dass sich die medianen Überlebenszeiten der Patienten mit metastasiertem, kastrationsresistenten Prostatakarzinom (mCRPC) dank wirksamer Therapiesequenzen deutlich verlängert haben. Der behandelnde Arzt müsse daher die Möglichkeiten der Sequenztherapie mit dem Patienten besprechen. Die systemische Sequenztherapie sei bei diesen Patienten längst klinische Realität, so Hammerer,.

Laut Hammerer zeigt die klinische Erfahrung, dass Abiraterone auch nach Cabazitaxel-Versagen wirksam ist, sodass nach Docetaxel-Versagen zunächst die Chemotherapie-Sequenz weitergeführt werden kann. Das biete sich vor allem bei Patienten an, die auf Docetaxel gut angesprochen haben oder für diejenigen, die einen schnellen PSA-Wert-Abfall oder eine schnelle Symptomlinderung benötigen beziehungswei- se wünschen. Bei der Therapieentscheidung müsse auch der Patientenwunsch berücksichtigt werden.

Darüber hinaus hat die klinische Erfahrung Cabazitaxel (Jevtana ${ }^{\circledR}$ ) als wirksame Therapieoption nach Docetaxel-Versagen weiter bestätigt. Cabazitaxel erreichte im direkten Phase-III-Vergleich der Zulassungsstudie (HR 0,$70 ; p<0,0001$ ) einen signifikanten Überlebensvorteil gegenüber Mitoxantron [de Bono JS et al. Lancet 2010, 376: 1147-54]. Anders als die meisten anderen neuen Substanzen wurde Cabazitaxel nicht gegen Placebo, sondern mit einem Verum verglichen. Die Sorge um limitierende Nebenwirkungen hat sich nach Aussage von Hammerer in der täglichen Routine nicht bestätigt. Voraussetzung sei jedoch ein aktives Nebenwirkungsmanagement und eine Patientenselektion, die sich an der Zulassung orientieren sollte. Viele Nebenwirkungen lassen sich so in ihrem
Schweregrad reduzieren oder ganz vermeiden. Hammerer erläuterte, dass Patienten unter Cabazitaxel nicht notwendigerweise primär prophylaktisch G-CSF (GranulozytenKolonie stimulierender Faktor) benötigen. Da das Neutropenierisiko in den ersten beiden Therapiezyklen am höchsten ist und danach deutlich abflacht, könne es jedoch besonders bei Risikopatienten durch die primär prophylaktische G-CSF-Gabe deutlich gesenkt werden. Er wies darauf hin, dass Cabazitaxel von vielen Patienten besser vertragen wird als Docetaxel; zum Beispiel stehen Poylneuropathien bei Cabazitaxel deutlich weniger im Vordergrund. Birgit-Kristin Pohlmann

Fortbildungsveranstaltung „Expertise Prostata", Potsdam, 24. März 2012; Veranstalter: Sanofi-Aventis, Frankfurt/Main

\section{Neue Phase-III-Daten: Kombinationstherapie verbessert Miktionsbeschwerden und Lebensqualität bei LUTS}

_ Im Rahmen der Jahrestagung der European Association of Urology (EAU) im Februar dieses Jahres in Paris wurden die Ergebnisse der zwölfwöchigen Phase-III-Studie NEPTUNE bei Männern mit Beschwerden der unteren Harnwege (Lower Urinary Tract Symptoms, LUTS) vorgestellt [Drake $\mathrm{M}$ et al. EAU 2012 Poster 746]. Die Patienten der Studie $(n=1.334)$ wurden in einer von vier Gruppen randomisiert: Placebo, Monotherapie mit Tamsulosin OCAS („oral controlled absorption system", 0,4 mg) oder eine Kombinationstherapie mit EC905 aus Tamsulosin OCAS $(0,4 \mathrm{mg})$ plus Solifenacin $(6 \mathrm{mg})$ beziehungsweise Tamsulosin OCAS $(0,4 \mathrm{mg})$ plus Solifenacin (9 mg).

EC905 ist eine fixe, einmal täglich oral einzunehmende Kombination aus Tamsulosin OCAS $^{\text {TM }}$ und Solifenacin $\left(\right.$ Vesikur $\left.^{\oplus}\right)$, die zur Behandlung von Entleerungs- als auch Speichersymptomen der LUTS bei Männern entwickelt wurde.

In allen drei Verumgruppen verminderte sich im Vergleich zu Placebo der Harndrang (Total Urgency Score) pro 24 Stunden ( $<<0,001$ ). Darüber hinaus war die Kombination aus
Tamsulosin OCAS $(0,4 \mathrm{mg})$ plus Solifenacin (6 mg) der Monotherapie mit Tamsulosin OCAS $(0,4 \mathrm{mg})$ signifikant überlegen $(p=0,025)$. Unter beiden Dosierungen der Kombinationstherapie und unter der Tamsulosin-Monotherapie verbesserte sich bei den Patienten der Internationale Prostata Symptomenscore im Vergleich zu Placebo (IPPS, $\mathrm{p}<0,05$ ); unter der Kombinationstherapie zeigten sich auch Verbesserungen in Bezug auf Miktionshäufigkeit, entleertes Volumen pro Miktion und Harndrang $(p<0,001)$. Tamsulosin OCAS plus Solifenacin $(6 \mathrm{mg}$ ) hatte ferner statistisch signifikante Vorteile gegenüber einer Tamsulosin-OCAS-Monotherapie $(0,4 \mathrm{mg})$ in Bezug auf Miktionshäufigkeit pro 24 Stunden und entleertes Volumen pro Miktion (beide $p<0,001$ ). Bei Patienten unter der EC905-Kombinationstherapie verbesserte sich der IPSS-Subscore zur Lebensqualität stärker als unter Placebo $(p<0,001)$ oder unter Tamsulosin OCAS allein $(p<0,05)$.

Patienten zeigten unter beiden Dosierungen der EC905-Kombinationstherapie statistisch signifikant stärkere Verbesserungen im IPSSSubscore zur Lebensqualität als jene unter
Placebo ( $p<0,001$ ) oder unter Tamsulosin OCAS allein $(p<0,05)$. Die Kombinationstherapie wurde gut vertragen. Behandlungsbedingte unerwünschte Ereignisse wie Mundtrockenheit traten bei 8,6\% der Patienten unter der niedriger dosierten Kombination beziehungsweise bei 10,5\% unter der höher dosierten Kombination auf, Obstipation bei $3,6 \%$ beziehungsweise $5,6 \%$.

„Die Ergebnisse der NEPTUNE-Studie machen deutlich, dass EC905 die Wirksamkeit im Vergleich zu Placebo beziehungsweise einer Tamsulosin-Monotherapie steigert - bei nur einer einzigen Tablette pro Tag", erklärte Prof. Marcus Drake, Bristol, der an der NEPTUNEStudie beteiligt war. „Bisher wurden den $\mathrm{Pa}$ tienten oft mehrere Tabletten verabreicht, um die Beschwerden erfolgreich zu behandeln. Eine Therapie, die bei anhaltender Effektivität und Verträglichkeit die Tablettenlast reduzieren kann, wird bei Patienten und Ärzten wahrscheinlich willkommen sein", so die Einschätzung von Drake.

Nach Informationen von

Astellas, München 